\title{
Potencial reprodutivo de touros Nelore acasalados coletivamente na proporção de um touro para 100 vacas
}

[Reproductive potential of Nellore bulls colectivelly mated with the ratio of one bull for each one hundred cows]

\author{
C. S. Franco ${ }^{1}$, V.O. Fonseca ${ }^{2}$, L. Gaste ${ }^{3}$ \\ ${ }^{1}$ Médico veterinário - Fazenda Jatobá - Brasilândia, MS \\ ${ }^{2}$ Escola de Veterinária - UFMG, Belo Horizonte, MG \\ ${ }^{3}$ Universidade Estadual de Londrina - Londrina, $\mathrm{Pr}$
}

\begin{abstract}
RESUMO
Utilizaram-se quatro touros selecionados por meio de exame andrológico (medida da circunferência escrotal e avaliação das qualidades físicas e morfológicas do sêmen) e de comportamento sexual (libido) que os classificaram entre muito bons e excelentes. Organizaram-se dois grupos experimentais, $G_{1}$ e $G_{2}$, o primeiro composto por dois touros e 200 vacas, estabelecendo, assim, a proporção de um touro para 100 vacas e o segundo, considerado como testemunha, por dois touros e 100 vacas, portanto, proporção de 1:50. Todas as matrizes eram da raça Nelore, pluríparas, não gestantes e secas. A estação de monta durou 72 dias. As taxas de gestação obtidas aos 30,60 e 72 dias, medidas a partir dos nascimentos das crias, foram de $54,0 \%$ e $57,0 \%(\mathrm{P}>0,05), 79,5 \%$ e $82,0 \%(\mathrm{P}>0,05)$ e $87,0 \%$ e $88,0 \%(\mathrm{P}>0,05)$, para os animais dos grupos $G_{1}$ e $G_{2}$, respectivamente. Reprodutores Nelore selecionados por meio de exames andrológicos e comportamentais (teste da libido) podem suportar até 100 vacas em estação de monta curta, com vantagens econômicas e para a eficiência reprodutiva atual e futura do rebanho, desde que observadas as regras de manejo pré-estabelecidas. A análise dos resultados revelou economia de $25,6 \%$ sobre o custo da cria desmamada em comparação com proporção simulada de 1:25 (4\% de touros) tradicionalmente utilizada na pecuária de corte brasileira.
\end{abstract}

Palavras-chave: bovino, Nelore, proporção touro: vacas, fertilidade

\begin{abstract}
Four sexually mature Nellore bulls evaluated for sexual soundness using physical and morphological characteristics of semen and scrotal circumference and sexual behavior (libido), were selected after qualifying either as very good or excellent. The chosen bulls were randomly divided into two experimental groups: group 1 - two bulls for mating 200 cows (1:100 ratio), and group 2 - two bulls for mating 100 cows (1:50 ratio). Females were pluriparous, opened, and non-nursing Nellore. Pregnancy rates on 30, 60 and 72 days during the breeding season, measured through birth date of the calves, were $54.0 \%$ vs. $57.0 \%(P>0.05), 79.5 \%$ vs. $82.0 \%(P>0.05)$, and $87.0 \%$ vs. $88.0 \%(P>0.05)$, for groups 1 and 2, respectively. The Nellore breeders selected through physical and morphological characteristics of semen, scrotal circumference and behavior (libido test) were able to bear up to 100 cows during a short breeding season, with economic advantage and genetic gain for the present and future reproductive efficiency of the herd, as long as pre-established management rules are properly followed. Economic analysis revealed a cost reduction of $25.6 \%$ of the weaned calves when using the proposed 1:100 bull/cow ratio instead of the traditional 1:25 ratio.
\end{abstract}

Keywords: cattle, Nellore, bull:cow ratio, fertility

Recebido em 4 de maio de 2005

Aceito em 2 de setembro de 2006

E-mail: jatoba@onda.com.br 


\section{INTRODUÇÃO}

A seleção de touros de alta eficiência reprodutiva é prática necessária para a obtenção de produtos melhorados e de custos mais baixos. Isto é possível quando se consegue a utilização de maior número de vacas por touro. No Brasil, esta proporção está, aleatoriamente, estabelecida em torno 1:25, o que exige elevado número de touros para o rebanho de, aproximadamente, 46,2 milhões de vacas e novilhas de corte em idade de reprodução. A conseqüência imediata, além do alto custo da cria desmamada, é a utilização de grande número de touros sem qualquer processo de seleção, oriundos, na maior parte das vezes, de lotes destinados ao abate. É de conhecimento geral que esta prática impede ou, pelo menos, retarda o processo evolutivo do rebanho de corte brasileiro, fundamentado na raça Nelore (Fonseca et al., 1997a, 2000; Pereira, 2004).

O potencial reprodutivo do touro é a soma de vários fatores inerentes à reprodução como a idade, puberdade, qualidade do sêmen, circunferência escrotal e libido, devidamente suportados por condição física adequada, de modo a assegurar o sucesso da monta e da fertilização (Fonseca et al., 1991). Esse potencial possui forte componente genético, sendo que a herdabilidade de algumas dessas características reprodutivas varia de média a alta, como a idade à puberdade e a circunferência escrotal (Valvasori et al., 1985; Bergmann et al., 1996; Pereira, 2004). Em outras características, entre elas a libido, a herdabilidade é pouco conhecida, embora apresente alta correlação com a capacidade de serviço e esta é de herdabilidade alta (0,59; Blockey, 1978). Assim, pelo que afirma Chenoweth (1983), pode-se induzir que a libido há de ser de herdabilidade alta, ou, pelo menos, média. Portanto, influências genéticas associadas às de meio, principalmente aquelas que têm maior influencia sobre o processo da espermatogênese, como as ligadas ao conforto térmico e à nutrição, determinam o potencial reprodutivo dos touros (Fonseca et al., 1992).

Esta pesquisa teve por objetivo avaliar o desempenho reprodutivo de touros Nelore, previamente selecionados, desafiados por nova proporção de touro por vacas de 1:100, em estação de monta natural curta e analisar os efeitos econômicos dessa proposição sobre o custo dos bezerros desmamados.

\section{MATERIAL E MÉTODOS}

Esta pesquisa foi desenvolvida na Fazenda Jatobá, no município de Brasilândia, MS, região típica de cerrado do Planalto Central Brasileiro, com inverno seco e relativamente frio e verão chuvoso e quente. A região é de uso restrito para pecuária de corte, mais especificamente, para atividade de cria. A gramínea predominante (90\% ou mais) é a Brachiaria humidicola.

Foram selecionados quatro touros para integrarem este experimento, entre os que obtiveram a maior pontuação nos exames andrológico e da libido. Foram organizados dois grupos, o primeiro $\left(\mathrm{G}_{1}\right)$ composto por dois touros e 200 vacas e o segundo $\left(\mathrm{G}_{2}\right)$, também por dois touros, porém, com 100 vacas. Dessa forma, obtiveram-se proporções touro:vacas de 1:100 e 1:50, respectivamente. $\mathrm{O} \mathrm{G}_{2}$ foi considerado grupo testemunha. As vacas eram da raça Nelore, todas pluríparas, não gestantes e secas. A estação de monta teve duração de 72 dias, iniciando-se em 15.11.2000 e terminando em 25.01.2001. Os touros foram submetidos ao exame clínico geral, à avaliação do seu escore corporal $(1=$ muito magro, $2=$ magro, $3=$ moderado, $4=$ gordo e 5 = muito gordo), ao exame andrológico completo com avaliação física e morfológica do sêmen e biometria testicular, segundo os critérios do CBRA (Fonseca et al., 1992b) e ao exame de comportamento sexual, pela avaliação da libido (Pineda et al., 1997). Para a obtenção do sêmen, utilizou-se o método da eletroejaculação, e a biometria testicular restringiu-se à mensuração da circunferência escrotal, segundo procedimentos descritos por Hahn et al. (1969) e Willet e Ohms (1975). Medidas de comprimento, largura, espessura e volume testiculares foram dispensadas por apresentarem alta correlação $(\mathrm{r}=$ 0,94) com a circunferência escrotal (Willet e Ohms, 1975).

De acordo com os resultados alcançados no exame andrológico, os touros foram classificados desde inaptos temporários até excelentes (Fonseca et al., 1997). Após terminado este exame, foram submetidos ao teste da libido, ou seja, avaliação do comportamento ou conduta sexual. Neste teste utilizaram-se vacas não gestantes e secas da raça Nelore cujos estros foram induzidos por aplicação de $5 \mathrm{ml}$ de $\mathrm{ECP}^{1}$,

${ }^{1}$ Upjhon - São Paulo, Brasil. 
via intramuscular profunda, ou pela associação de $5 \mathrm{ml}$ de ECP e $3 \mathrm{ml}$ de prostaglandina, também via intramuscular, naquelas que, pelo exame dos ovários, via palpação transretal, apresentavam corpo lúteo funcional. Os testes da libido foram realizados em currais de, aproximadamente, $400 \mathrm{~m}^{2}$. Para cada touro foram oferecidas duas ou três vacas em estro, além de outras duas ou três fora de estro para servirem de companheiras e tornarem o ambiente do teste mais natural. Todas foram expostas aos touros até apresentarem fadiga devido ao excesso de montas que tiveram de suportar, ou manifestarem sinais de final de estro. A duração do teste de cada touro, individualmente, foi de 10 minutos e, de acordo com o desempenho apresentado que variou desde o total desinteresse pela vaca até duas ou mais coberturas dentro do período (notas 0 e 10, respectivamente), foram selecionados quatro, classificados como excelentes e muito bons e que também já haviam alcançado ótima classificação no exame andrológico. Para se obter a proporção de vacas por touro, utilizou-se o esquema proposto por Fonseca (2000), que consistiu na associação entre o exame andrológico e o da libido.

Os testes de fertilidade a campo foram realizados em duas áreas de características semelhantes. Os dois touros do $G_{1}$ foram colocados com 200 vacas em pasto com área de 311,20ha, inteiramente formado com Brachiaria humidícola. Os do $\mathrm{G}_{2}$ permaneceram em pasto contíguo, com área de 241,73ha, formado com a mesma gramínea. Ambos os pastos continham bebedouros do tipo australiano e cochos para suplementação mineral ad libitum. Com o objetivo de facilitar o encontro dos parceiros sexuais, estabeleceu-se o manejo de "parar rodeio", que constituiu de visitas diárias na primeira semana e a cada dois dias a partir da segunda semana, na parte da manhã (até oito horas), ao local do experimento, momento em que todo o rebanho era reunido em um ponto do pasto que, uma vez determinado, não se alterou até o final da pesquisa. O mês de concepção da vaca foi determinado pelo mês de nascimento da cria, considerando-se um período de gestação médio de 290 dias. Assim, para nascimentos observados entre 3 de setembro e 30 de setembro de 2001, estimaram-se concepções ocorridas entre 15 de novembro e 14 de dezembro de 2000. Para nascimentos entre 1 de outubro e 31 de outubro de 2001, concepções ocorridas entre 15 de dezembro de 2000 e 14 de janeiro de 2001, e para nascimentos entre 1 de novembro e 18 de novembro de 2001, concepções ocorridas entre 15 de janeiro e 25 de janeiro de 2001 .

A análise estatística consistiu na comparação das taxas de gestação observadas aos 30, 60 e 72 dias para os animais dos grupos $\mathrm{G}_{1}$ e $\mathrm{G}_{2}$ pelo teste do qui-quadrado $\left(x^{2}\right)$.

Para avaliação dos custos das diferentes proporções de touro por vacas utilizadas $[(1: 25$, simulado), 1:50 e 1:100, observados] foi aplicada a equação de Fonseca et al. (1997):

$\mathrm{Y}=[(\mathrm{tt} \cdot \mathrm{c}) \cdot \mathrm{rc}+(\mathrm{tr} \cdot \mathrm{c}-\mathrm{ta} \cdot \mathrm{va})+(\mathrm{tt} . \mathrm{cm})]-\mathrm{cr}, \mathrm{em}$ que:

$\mathrm{Y}$, custo do modelo aplicado

$\left(\mathrm{Y}_{\mathrm{t}, \text { tradicional }}=1: 25\right.$ e $\mathrm{Y}_{\mathrm{p}}$, proposto $=1: 100, \mathrm{ou}$ outro);

$\mathrm{Tt}=$ total de touros utilizados no sistema ( $\mathrm{t}$ ou $\mathrm{p})$; $\mathrm{c}=$ capital (custo de um touro);

$\mathrm{rc}=$ remuneração do capital (aproximadamente $12 \%$ ao ano);

$\operatorname{tr}=$ total de touros adquiridos para reposição;

ta $=$ total de touros vendidos para abate;

$\mathrm{va}=$ valor de um touro abatido;

$\mathrm{cm}=$ custo de manutenção anual de um touro;

$\mathrm{cr}=$ capital residual (valor do lote dos touros em uso).

O custo de cada touro foi estabelecido em CR\$ $4.200,00$, valor de um reprodutor de 24 meses praticado nos leilões da região. A reposição de touros foi considerada como sendo de $20 \%$ e o descarte para abate por causas diversas, de $20 \%$ a um preço de CR\$ 1.025,00. O custo anual de manutenção de cada touro foi estabelecido em CR\$ 310,00 (valor médio de uma cria desmamada que seria obtida em seu lugar) e o valor residual dos touros que permaneceram no rebanho de CR\$ $3.500,00$. A taxa de desmama foi de $80 \%$ e o preço médio da comercialização das crias (machos e fêmeas) de CR \$ 310,00 por unidade.

Pela manipulação destes dados a partir da equação utilizada, chegou-se à economia de $25,6 \%$ entre a proporção simulada de 1:25 e a pesquisada de 1:100 e a $8,7 \%$ entre as proporções pesquisadas de 1:50 (lote testemunha) e 1:100 (lote desafiado), economia esta sempre a favor da proporção de $1: 100$.

Os prováveis resultados da proporção de 1:25 foram simulados com o objetivo de comparar custos com as proporções estudadas, uma vez que é 
amplamente utilizada na pecuária de corte nacional (Fonseca et al., 1997a, 2000; Pereira, 2004).

\section{RESULTADOS E DISCUSSÃO}

A classificação obtida pelos touros deste experimento, avaliados pelos exames andrológico e da libido, encontra-se na Tab. 1 bem como as proporções de vacas por touro recomendada e testada. A eficiência reprodutiva de cada grupo experimental, avaliada pelas taxas de concepção aos 30, 60 e 72 dias de estação de monta, tendo por base o mês de nascimento da cria, está na Tab. 2, e a eficiência reprodutiva avaliada pelos partos quinzenais, na Tab.3.

Tabela 1. Classificação dos touros Nelore após avaliação pelos testes andrológico (teste 1) e da libido (teste 2 ) e proporções testadas e recomendadas do número de vacas

\begin{tabular}{|c|c|c|c|c|c|c|}
\hline \multirow{2}{*}{ Grupo } & \multirow{2}{*}{$\begin{array}{l}\mathrm{N}^{\circ} \text { do } \\
\text { touro }\end{array}$} & \multirow{2}{*}{$\begin{array}{c}\text { Idade } \\
\text { (meses) }\end{array}$} & \multicolumn{2}{|c|}{ Classificação } & \multirow{2}{*}{$\begin{array}{l}\text { Proporção } \\
\text { recomendada }\end{array}$} & \multirow{2}{*}{ Proporção testada } \\
\hline & & & Teste 1 & Teste 2 & & \\
\hline \multirow{2}{*}{$\mathrm{G}_{1}$} & K-90 & 60 & Excelente & Muito Bom & $1: 70$ & \multirow{2}{*}{$1: 100$} \\
\hline & K-94 & 60 & Excelente & Excelente & $1: 80$ & \\
\hline \multirow{2}{*}{$\mathrm{G}_{2}$} & $\mathrm{~K}-72$ & 60 & Excelente & Excelente & $1: 80$ & \multirow{2}{*}{$1: 50$} \\
\hline & $\mathrm{K}-120$ & 72 & Excelente & Excelente & $1: 80$ & \\
\hline
\end{tabular}

${ }^{1}$ Fonseca et al. (1997b). G1 = grupo experimental composto por 200 vacas e dois touros; G2 = grupo testemunha, composto por 100 vacas e dois touros.

Tabela 2. Taxas de concepção de vacas Nelore em estação de monta de 72 dias, avaliadas tendo por base o dias após nascimento da cria

\begin{tabular}{|c|c|c|c|c|c|c|c|c|}
\hline \multirow{3}{*}{ Grupo } & \multirow{3}{*}{$\begin{array}{l}\mathrm{N}^{0} \text { de } \\
\text { vacas }\end{array}$} & \multirow{3}{*}{$\begin{array}{l}\mathrm{N}^{\mathrm{o}} \mathrm{de} \\
\text { touros }\end{array}$} & \multicolumn{6}{|c|}{ Taxa de gestação } \\
\hline & & & \multicolumn{2}{|c|}{30 dias } & \multicolumn{2}{|c|}{60 dias } & \multicolumn{2}{|c|}{72 dias } \\
\hline & & & $\mathrm{N}$ & $\%$ & $\mathrm{n}$ & $\%$ & $\mathrm{n}$ & $\%$ \\
\hline $\mathrm{G}_{1}$ & 200 & 2 & 108 & 54,0 & 159 & 79,5 & 174 & 87,0 \\
\hline $\mathrm{G}_{2}$ & 100 & 2 & 57 & 57,0 & 82 & 82,0 & 88 & 88,0 \\
\hline Total & 300 & 4 & 165 & 55,0 & 241 & 80,3 & 262 & 87,3 \\
\hline
\end{tabular}

$\mathrm{G}_{1}=$ proporção touro:vaca de 1:100 (experimental); $\mathrm{G}_{2}=$ proporção touro:vaca de 1:50 (testemunha). Diferença entre grupos não significativa $(\mathrm{P}>0,05)$.

Tabela 3. Número e percentagem de bezerros nascidos das vacas Nelore durante a estação de nascimentos de setembro a novembro

\begin{tabular}{|c|c|c|c|c|c|c|c|c|c|c|c|c|}
\hline \multirow{3}{*}{ Período } & \multicolumn{4}{|c|}{ Setembro } & \multicolumn{4}{|c|}{ Outubro } & \multicolumn{4}{|c|}{ Novembro } \\
\hline & \multicolumn{2}{|c|}{$\mathrm{G}_{1}$} & \multicolumn{2}{|c|}{$\mathrm{G}_{2}$} & \multicolumn{2}{|c|}{$\mathrm{G}_{1}$} & \multicolumn{2}{|c|}{$\mathrm{G}_{2}$} & \multicolumn{2}{|c|}{$\mathrm{G}_{1}$} & \multicolumn{2}{|c|}{$\mathrm{G}_{2}$} \\
\hline & $\mathrm{n}$ & $\%$ & $\mathrm{n}$ & $\%$ & $\mathrm{n}$ & $\%$ & $\mathrm{n}$ & $\%$ & $\mathrm{n}$ & $\%$ & $\mathrm{n}$ & $\%$ \\
\hline $1^{\mathrm{a}}$ Quinzena & 57 & 32,76 & 25 & 28,41 & 38 & 21,84 & 20 & 22,73 & 13 & 7,47 & 6 & 6,82 \\
\hline $2^{\mathrm{a}}$ Quinzena & 51 & 29,31 & 32 & 36,36 & 13 & 7,47 & 5 & 5,68 & 2 & 1,15 & 0 & 0 \\
\hline Totais & 108 & 62,07 & 57 & 64,77 & 51 & 29,31 & 25 & 28,41 & 15 & 8,62 & 6 & 6,82 \\
\hline
\end{tabular}

$\mathrm{G}_{1}=$ grupo experimental composto por 200 vacas e dois touros; $\mathrm{G}_{2}=$ grupo testemunha composto por 100 vacas e dois touros.

Todos os quatro touros apresentavam alto potencial reprodutivo (Tab. 1), o que os credenciava para atender a até 70 (touro K-90) e 80 (demais touros) vacas durante a estação de monta, normalmente variando de 90 a 120 dias, conforme recomendação de Fonseca et al. (2000). Entretanto, os dois do G1 foram desafiados a servir 100 vacas cada um em estação de monta com duração máxima de 72 dias.

As taxas de concepção, obtidas pelas vacas dos grupos experimental e testemunha, não divergiram entre si (Tab. 2), embora se esperasse que a elevada proporção de 100 vacas por touro e, ainda, em estação de monta mais curta que as recomendadas (Fonseca et al., 2000), os 
exaurisse e, conseqüentemente, determinasse menor taxa de fertilidade das vacas por eles servidas. Estes resultados demonstraram que o limite da capacidade reprodutiva do touro Nelore ainda não foi atingida e foram próximos daqueles verificados por Fonseca et al. (1997a, 2000), que obtiveram $53,5 \%$ e $64,6 \%$ aos 30 dias e $94,2 \%$ e $95,5 \%$, ao final das estações de monta de 120 e 90 dias, com proporções de 40 e 60 e 40 e 80 vacas por touro, respectivamente, por Silva (1994) que assinalou taxa de fertilidade de $92,8 \%$ ao final da temporada de 90 dias, ao trabalhar com proporções de 60 vacas por touro, e por Galvani (1998), que verificou taxa de fertilidade de $60,6 \%$ e $90,9 \%$ aos 21 e 63 dias, respectivamente, ao trabalharem com até 96 vacas por touro.

Os partos distribuíram-se de modo semelhante entre G1 e G2 ao longo da estação de nascimento, revelando grande número de vacas gestantes nos primeiros dias da estação de monta (Tab. 3), o que indica confiabilidade nos touros de potencial reprodutivo (PR) superior, pois, apesar de desafiados pelo elevado número de vacas em estro, mostraram-se competentes, permitindo que a maior parte delas concebessem no primeiro terço da estação de monta. A concepção precoce dentro da estação de monta é considerada como um dos fatores mais importante para a alta eficiência reprodutiva do rebanho (Ribeiro, 1993; Fonseca et al., 1997a, 2000).

Fatores físicos como a área e a topografia das pastagens, barreiras como bosques, elevações e outras citadas por Ribeiro (1993) que pudessem dificultar o encontro dos touros com as vacas em estro foram minimizados neste trabalho, conforme descrição das pastagens, que, embora extensas, estavam bem formadas, além de utilizar-se o eficiente manejo de "parar rodeio" para facilitar o encontro dos parceiros sexuais. É importante frisar que, embora o número de vacas em estro no início da estação de monta tenha sido elevado, o que se conclui pela observação da Tab. 3 (mais de 50\% de nascimentos no primeiro mês, portanto, resultantes de cobrições realizadas nos primeiros 30 dias da estação de monta), não houve quaisquer interferências negativas sobre as taxas de concepção que pudessem ser imputadas à hierarquia social. Embora essa hierarquia seja rigorosa na organização social dos bovinos, o grande número de fêmeas a serem cobertas no início da estação de monta e uma possível exaustão dos touros no seu final contribuíram para a não interferência dos dominantes sobre os vassalos no sentido de impedi-los de cobrir vacas em estro. A metodologia aqui desenvolvida e os resultados obtidos permitem, então, a utilização de acasalamentos coletivos (mais de um touro e suas vacas na mesma pastagem) com elevada proporção de vacas por touro, o que viabiliza o método para as grandes fazendas de criar do Planalto Central Brasileiro, conforme também verificado por Fonseca et al. (1997a, 2000), Pineda e Fonseca (1999), e na Planície Amazônica por Galvani (1998).

As economias no custo das crias, de 25,6\% observada neste experimento entre as proporções de 1:100 (pesquisada) e 1:25 (simulada) e de $8,7 \%$ entre as proporções de 1:100 e 1:50 (grupo testemunha) foram superiores às relatadas por Fonseca et al. (1997), de 18,6\%, e de Fonseca et al. (2000), de 22,8\%, ao analisarem os custos obtidos entre proporções de 60 e 25 e 80 e 25 vacas por touro, respectivamente. Essas diferenças se explicam pela proporção mais elevada de vacas deste experimento e pelo diferencial de preço de touros melhoradores da raça Nelore, os mais indicados para a pecuária brasileira. A maior economia no presente experimento justifica a afirmativa de que quanto menor a percentagem de touros em relação ao número de vacas do rebanho, menor o custo da cria desmamada (Fonseca et al., 2000).

\section{CONCLUSÕES}

Os resultados mostram que os touros Nelore vêm sendo subutilizados no rebanho brasileiro, uma vez que são eficientes para cobrirem até 100 fêmeas em estação de monta curta, quando de elevado potencial reprodutivo. Demonstram, ainda, que reprodutores previamente selecionados, a exemplo dos que se utilizaram neste trabalho, podem ser recomendados para a elevação do patamar de 25 vacas por touro, prática enraizada na pecuária de corte nacional, para a média de 50 delas por reprodutor, sem prejuízo da fertilidade e com vantagens sobre o custo do bezerro desmamado e para o melhoramento genético do rebanho, devido ao fato de se permitir aos melhores touros a paternidade do maior número dos bezerros 
nascidos. Finalmente, demonstra-se que o limite do potencial reprodutivo do touro Nelore ainda não foi atingido.

\section{REFERÊNCIAS BIBLIOGRÁFICAS}

BERGMANN, J.A.G.; ZAMBORLINI, L.C.; PROCÓPIO, C.S.O. et al. Estimativas de parâmetros genéticos do perímetro escrotal e do peso corporal em animais da raça Nelore. Arq. Bras. Med. Vet. Zootec., v.48, p.69-78, 1996.

BLOCKEY, M.A.B. Heritability of serving capacity and scrotal circumference in beef bulls. Am. Soc. Anim. Sci., 1978. (Abstract n.92)

CHENOWETH, P.J. Sexual behavior of the bull: a review. J. Dairy Sci., v.66, p.173-179, 1983.

FONSECA, V.O. O touro no contexo da eficiência reprodutiva do rebanho. Inf. Agropec., v.21, p.48-63, 2000.

FONSECA, V.O.; CRUDELI, G.A.; COSTA E SILVA, E.V. et al. Aptidão reprodutiva de touros da raça Nelore: efeito de diferentes estações do ano sobre as características seminais, circunferência escrotal e fertilidade. Arq. Bras. Med. Vet. Zootec., v.44, p.7-15, 1992a.

FONSECA, V.O.; CRUDELI, G.A.; COSTA E SILVA, E.V. et al. Potencial reprodutivo de touros da raça Nelore (Bos taurus indicus) em monta natural. Proporção touro:vaca 1:40 e fertilidade. Rev. Bras. Reprod. Anim., v.15, p.103-108, 1991.

FONSECA, V.O.; FRANCO, C.S.; BERGMANN, J.A.G. et al. Potencial reprodutivo de touros da raça Nelore (Bos taurus indicus) acasalados com elevado número de vacas. Arq. Bras. Med. Vet. Zootec., v.49, p.5362, 1997a.

FONSECA, V.O.; FRANCO, C.S.; BERGMANN, J. A. G. Potencial reprodutivo de touros Nelore em monta natural. Proporção touro:vaca 1:80 em acasalamentos coletivos. Taxa de fertilidade e aspectos econômicos. Arq. Bras. Med. Vet. Zootec., v.52, p.77-82, 2000.

FONSECA, V.O.; SANTOS, N.R.; MALINSKI, P.R. Classificação andrológica de touros zebus com base no perímetro escrotal e características morfológicas do sêmem, Rev. Bras. Reprod. Anim., v.21, p.36-39, 1997 b.

FONSECA, V.O.; VALE FILHO, V.R.; MIES FILHO, A. et al. Procedimentos para exame andrológico e avaliação de sêmen animal. Belo Horizonte: CBRA, 1992b. 79p.

GALVANI, F., Desempenho reprodutivo de touros de alta libido da raça Nelore. 1998. $70 \mathrm{f}$. Dissertação (Mestrado) - Departamento de Medicina Veterinária, Universidade Federal de Viçosa, Viçosa, MG.

HAHN, J.; FOOT, R.H.; SEIDEL, G.E. Testicular growth and related sperm output in dairy bulls. J. Anim. Sci., v.29, p.41-47, 1969.

PEREIRA, J.C.C. Melhoramento genético aplicado à produção animal, Belo Horizonte: FEPMVZ-Editora, 2004. 609 p.

PINEDA, N.R.; FONSECA, V.O. JORNADAS DE ACTUALIZÁCION DE LA ASOCIACIÓN DE CRIADORES DE CEBU. 1. 1999, Caracas. Caracas, Venezuela: Memorias, 1999. p. 27-39.

PINEDA, N.R.; LEMOS, P.F.; FONSECA, V.O. Comparação entre dois testes de avaliação do comportamento sexual (libido de touros Nelore) (Bos taurus indicus). Rev. Bras. Reprod. Anim., v. 21, p. 29-34, 1997.

RIBEIRO, W.N. Aplicação do teste de capacidade de serviço na avaliação da saúde reprodutiva de touros europeus. In: CONGRESSO BRASILEIRO DE REPRODUÇÃO ANIMAL, 10., 1993, Belo Horizonte. Anais... Belo Horizonte: CBRA, 1993. p.77-84.

SILVA, E.V.C. Capacidade reprodutiva de touros Nelore: exame andrológico, teste de comportamento sexual e desafio de fertilidade. 1994. 102f. Dissertação (Mestrado) - Escola de Veterinária da Universidade Federal de Minas Gerais, Belo Horizonte.

VALVASORI, E.; TROVO, J.B.; PROCKNOR, M. et al. Biometria testicular em tourinhos Gir, Guzerá, Nelore e Caracu. Bol. Ind. Anim., v.42, p.155-166, 1985.

WILLET, E.L.; OHMS, J.I. Measurement of testicular size and its relations to production of spermatozoa by bulls. J. Dairy Sci., v.40, p.15591569, 1975. 